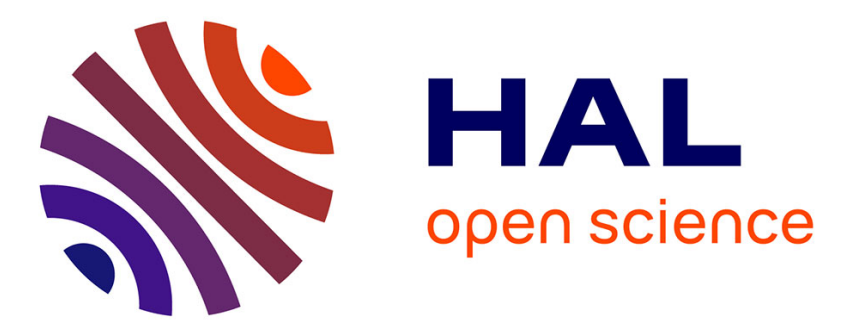

\title{
Finger kunckcle patterns based person recognition via bank of multi-scale binarized statistical texture features
}

\author{
Abdelouahab Attia, Mourad Chaa, Zahid Akhtar, Youssef Chahir
}

\section{To cite this version:}

Abdelouahab Attia, Mourad Chaa, Zahid Akhtar, Youssef Chahir. Finger kunckcle patterns based person recognition via bank of multi-scale binarized statistical texture features. Evolving Systems, 2020, 11 (4), pp.625-635. hal-01956894

\section{HAL Id: hal-01956894 \\ https://hal.science/hal-01956894}

Submitted on 16 Dec 2018

HAL is a multi-disciplinary open access archive for the deposit and dissemination of scientific research documents, whether they are published or not. The documents may come from teaching and research institutions in France or abroad, or from public or private research centers.
L'archive ouverte pluridisciplinaire HAL, est destinée au dépôt et à la diffusion de documents scientifiques de niveau recherche, publiés ou non, émanant des établissements d'enseignement et de recherche français ou étrangers, des laboratoires publics ou privés. 


\title{
Finger kunckcle patterns based person recognition via bank of multi- scale binarized statistical texture features
}

\author{
Abdelouahab Attia ${ }^{1} \cdot$ Mourad Chaa $^{2} \cdot$ Zahid Akhtar $^{3} \cdot$ Youssef Chahir $^{4}$
}

\begin{abstract}
This paper proposes a novel finger knuckle patterns (FKP) based biometric recognition system that utilizes multi-scale bank of binarized statistical image features (B-BSIF) due to their improved expressive power. The proposed system learns a set of convolution filters to form different BSIF feature representations. Later, the learnt filters are applied on each FKP traits to determine the top performing BSIF features and respective filters are used to create a bank of features named B-BSIF. In particular, the presented framework, in the first step, extracts the region of interest (ROI) from FKP images. In the second step, the B-BSIF coding method is applied on ROIs to obtain enhanced multi-scale BSIF features characterized by top performing convolution filters. The extracted feature histograms are concatenated in the third step to produce a large feature vector. Then, a dimensionality reduction procedure, based on principal component analysis and linear discriminant analysis techniques (PCA + LDA), is carried out to attain compact feature representation. Finally, nearest neighbor classifier based on the cosine Mahalanobis distance is used to ascertain the identity of the person. Experiments with the publicly available PolyU FKP dataset show that the presented framework outperforms previously-proposed methods and is also able to attain very high accuracy both in identification and verification modes.
\end{abstract}

Keywords Biometric $\cdot$ Image local descriptor $\cdot$ BSIF $\cdot$ Dimensionality reduction · Classification

\section{Introduction}

Abdelouahab Attia

attia.abdelouahab@gmail.com

Mourad Chaa

chaawissam92@gmail.com

Zahid Akhtar

zmomin@memphis.edu

Youssef Chahir

youssef.chahir@unicaen.fr

1 Computer Science Department, Faculty of Mathematics and Informatics, University of Mohamed El Bachir El Ibrahimi, 34000 Bordj Bou Arreridj, Algeria

2 Lab. ELEC, Faculty of New Technology of Information and Communication, Ouargla University, 30000 Ouargla, Algeria

3 University of Memphis, Memphis, USA

4 Lab. Image Team GREYC-CNRS UMR 6072 University of Caen, Caen, France
In today's highly interconnected society, automated personal identification techniques have become vital of security systems, which require high classification schemes (Angelov and Gu 2018; Bao et al. 2018). One such person recognition technique is biometrics, that is identifying a person based on their physiological and behavioral characteristics (Adeoye 2010). Biometrics have several advantages compared to conventional methods such as passwords or ID cards, e.g., it can be forgotten or lost (Adeoye 2010; Rani and Shanmugalakshmi 2013; Akhtar and Alfarid 2011). In fact, many biometric traits have been proposed and widely being accpeted as well as used in diverse applications ranging from ranging from border crossing to mobile authentication (Zhang et al. 2018b; Akhtar et al. 2011). Recently, FKP characteristics is one of the hand based biometrics that is prominent emerging trait owing to advantages: (1) the acquisition of fingerknuckle surfaces is relatively easy with low cost and lowresolution simple cameras, (2) FKP based access systems can be used under various environmental conditions such as indoor and outdoor, (3) FKP features are stable and remain 
unchanged over time, i.e., FKP patterns are age-invariant, (4) FKP based biometric recognition systems are very reliable that gives high performances (Rani and Shanmugalakshmi 2013).

In the literature, few methods for FKP based biometric recognition systems have been proposed. For instance, Zhang et al. (2011) have presented an algorithm that integrated local orientations via ensemble of Gabor filters and global orientations via Fourier coefficients features for FKP person authentication. While, Zhang and Li (2012) enhanced the FKP recognition accuracy using two Riesz-transformbased coding schemes (i.e., RCode1 and RCode2). Shariatmadar and Faez (2013) have proposed a FKP recognition scheme usable both in personal identification and verification modes. Specifically, the scheme divides the ROI of captured image into a set of blocks and Gabor filters are applied on each block, which is followed by binary patterns extraction as feature histograms. Lastly, Bio hashing method is employed for matching process between the template and query histograms. Zeinali et al. (2014) proposed to use Directional Filter Bank (DFB) feature extraction with dimensionality reduction by LDA method for FKP recognition. Authors in (Shariatmadar and Faez 2014) devised a competent method based on multi-instance FKP fusion, which integrates the information from diverse fingers [i.e., he left index/middle fingers (LIF, LMF) and right index/ middle fingers (RIF, RMF)]. The information are fused at different level, i.e., feature-, matching scores- and decisionlevels.Nigam et al.(Nigam et al. 2016) introduced a FKP based authentication system that depends on multiple textures with score level fusion. Firstly, FKP images are preprocessed by Gabor filters. Secondly, quality parameters are extracted to be later made use of in recognition procedure. Tao et al. (Kong et al. 2014) designed a hierarchical classification method for FKP recognition. The system consists of two stages. In the first stage, recognition is conducted using basic Gabor feature and a novel decision rule with the fixed threshold. Whereas, in the second stage, speeded-up robust feature is used only when the basic feature is not able to perform recognition successfully. In turn, Chaa et al.(Chaa et al. 2018) introduced a novel method that combines two types of the histograms of oriented gradients (HOG) extracted from reflectance and illumination FKP trait by Adaptive Single Scale Retinex (ASSR) algorithm. While, a novel reconstruction-based finger-knuckle-print (FKP) verification method to decrease the false rejections caused by finger pose variations in data capturing process is devised by Zhang et al. (2018a, b). Recently, Chlaoua et al. (2018) have employed a simple deep learning method known as principal component analysis Network (PCANet). In the proposed model, PCA has been employed to learn two-stages of filter banks then a simple binary hashing and block histograms for clustering of feature vectors are used. The resultant features are finally fed to classification step, i.e., linear multiclass Support Vector Machine (SVM). The authors also studied a multimodal biometric system based on matching score level fusion. Zhai et al. (2018) designed a FKP recognition system using batch normalized convolution neural network with random histogram equalization as data augmentation scheme.

All in all, despite FKP being easy to use trait for person recognition, comparatively very limited researches have been conducted on FKP biometrics. Moreover, majority of existing FKP methods are not able to attain very high accuracy due to use of features that have lower power of discrimination. Similarly, they are very sensitive to image orientations and scales. Therefore, in this paper, we propose an automated method for finger knuckle pattern recognition that can attain excellent accuracy. The presented framework extracts ROIs from the capture finger knuckle images, which is followed by application of BSIF image descriptor on each ROI. BSIF is very robust to rotation and illumination variations. Then, the best features (corresponding to BSIF convolution filters) that achieve higher performances are selected to construct a bank of BSIF image representations. Next, a PCA + LDA based dimensionality reduction scheme is employed to obtain compact representation of the FKP trait. Lastly, nearest neighbor classifier is utilized to authenticate the user.

The remainder of this paper is organized as follows: In Sect. 2, the proposed system is described including feature extraction and matching process used in the system. Experimental protocol, dataset, and figures of merit are presented, and experimental results are given in Sect. 3. Finally, the conclusion and further works are drawn in Sect. 4.

\section{Proposed FKP biometric system}

Figure 1 depicts the schematic diagram of the proposed finger knuckle pattern (FKP) based person recognition system. The framework consists of two phases: enrollment and identification. During enrollment phase, each user presents their finger knuckle to the sensor. The FKP image of the user is captured and processed to produce regions of interest described in Sect. 2.1. Then, the features are extracted using B-BSIF method, which is detailed in Sect. 2.2. The top performing extracted features from ROI are concatenated to form raw histogram (long feature vector) on which a PCA + LDA dimensionality reduction technique discussed in Sect. 2.3 is applied to obtain concise feature representation. The compact feature representation is stored in the database as template. All templates of enrolled users are used as training data for decision making classifier explained in Sect. 2.4. During verification phase, the captured FKP image from the user undergoes the above-mentioned process, and this time 
Fig. 1 Proposed framework for person recognition using FKP (finger knuckle print)

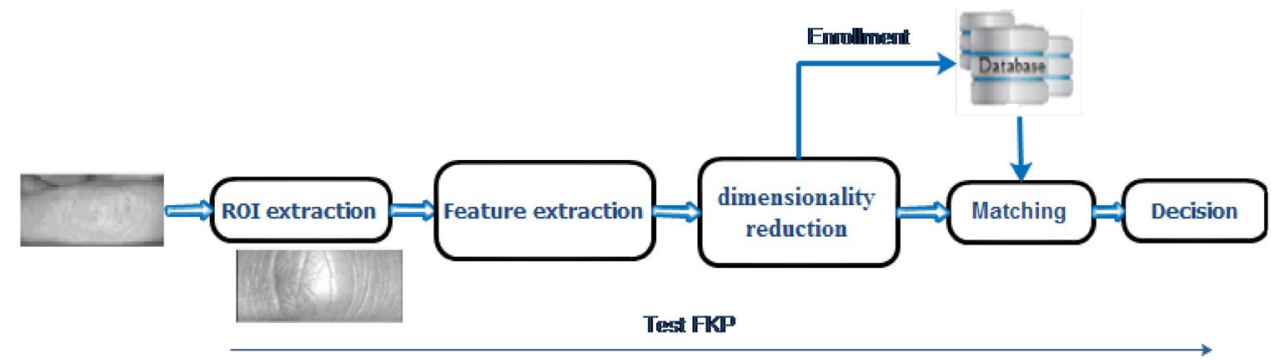

the extracted features are fed to the decision-making classifier to determine the identity of the person.

\subsection{Extractionof the region of interest (ROI)}

The procedure of extrcating the region of interest from FKP images are similar to the method presented in (Zhang et al. 2010).The ROI extraction technique is composed of four steps, which are explanied as follows:

Step 1: A Gaussian smoothing process is applied on the original FKP image, which is then down-sampled as the smoothed image to150 dpi (Dots Per Inch).

Step 2: The X-axis of the coordinate system is fixed so that the bottom boundary of the finger can be easily extracted. The bottom boundary of the finger is determined by a canny edge detector.

Step 3: The Y-axis of the coordinate system is determined by applying a Canny edge detector on the cropped sub-image extracted from the original FKP image based on $\mathrm{X}$-axis. This process helps finding the convex direction coding scheme.

Step 4: The ROI regions indicated by the rectangle are extracted.

Figure 2 illustrates the global steps the of ROI extraction.

\subsection{Binarized statistical image features extraction}

Binarized Statistical Image Features (BSIF) is a local image descriptor constructed by binarizing the responses to linear filters (Kannala and Rahtu 2012). BSIF learns a set of convolution filters from natural images using ICA (Independent Component Analysis) based on unsupervised scheme. These learned filters are used to represent each pixel of the capture FKP image as a binary string by computing its response to learned convolution filters. The binary string for each pixel can be considered as a local descriptor of the image intensity pattern in the neighborhood of that pixel. Finally, the histogram of the pixels binary string values allows one to characterize the texture properties within the image sub-regions.

In this paper, we have used the open-source filters (Kannala and Rahtu 2012), which were trained using 50,000 image patches randomly sampled from 13 different natural scenic images. Three main steps build the BSIF filters: mean subtraction of each patches, dimensionality reduction using PCA (Principle Component Analysis), and estimation of statistically independent filters (or basis) using ICA. Give a FKP image sample $I$ of size $\mathrm{n} \times \mathrm{m}$ and a filter $F_{i}$ of same size, filter response is attained as follows (Kannala and Rahtu 2012):

$\mathrm{r}_{i}=\sum_{\mathrm{n}, \mathrm{m}} I(n, m) F_{i}(n, m)$

where $F_{i}$ stands for the convolution filters $i=\{1,2, \ldots, \mathrm{m}\}$ that represent statistically independent filters whose responses can be calculated simultaneously and then binarized in order to obtain the binary string as follow(Kannala and Rahtu 2012):

$\mathrm{b}_{i}=\left\{\begin{array}{cc}1 & \text { if } \mathrm{r}_{i}>0 \\ 0 & \text { otherwise }\end{array}\right.$

Finally, the BSIF features are obtained as a histogram of pixel's binary codes that can efficiently describe the texture components of the FKP image. There are two essential factors into BSIF descriptor explicitly: the filter sizeand the length of the filter $(i)$. Single filters with a fixed length may not be capable of generalizing well the finger knuckle patterns with varying intensities, scale and orientations. Therefore, we propose to utilize high performing multiple filters

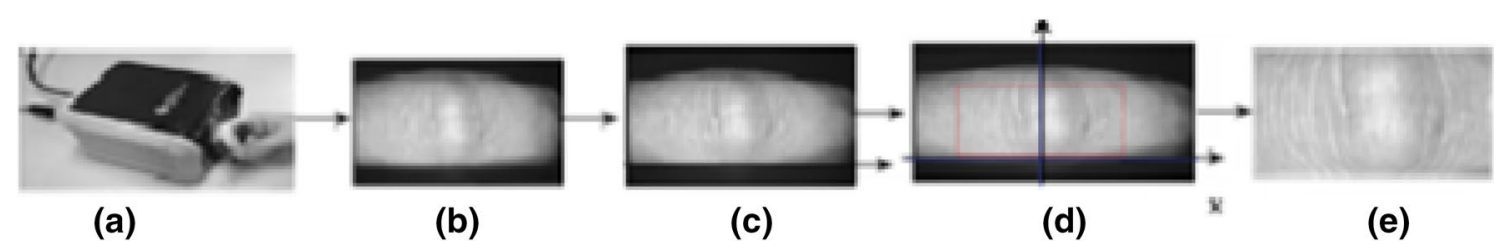

Fig. 2 Steps involved in ROI extraction of FKP image 
with different scales in order to capture eminent features, thus the name bank of binarized statistical image features (B-BSIF), which are further detailed in experimental section.

Figure 3 shows an example of an FKP image and the BSIF filters processing. Figure 3 a presents the input ROI FKP image. Figure $3 \mathrm{~b}$ illustrates the learned BSIF filter with a size $11 \times 11$ and of length 12 bits. While, Fig. $3 c$ depicts the results of the individual convolution of the ROI FKP image with respective BSIF filters. Figure $3 d$ presents the final BSIF encoded feature/image.

\subsection{Dimensionality reduction}

The histograms extracted from the encoded FKP image using B-BSIF descriptor are merged into one big feature vector. These vectors are big with high dimensions. Thus, it becomes difficult to process and evaluate them. Therefore, we perform dimensionality reduction before matching. The Principal Component Analysis (PCA) is a widely and simple method for dimensionality reduction, but in PCA separability between classes is ignored (Turk and Pentland 1991). To avoide PCA's issue and to achieve more improved separability of feature subspace, Linear Discriminative Analaysis (LDA) (Belhumeur et al. 1997) may be deployed that may lead to attractive performances for recognition tasks. Though LDA still assumes a common covariance matrix among the classes that violates the normality principle. To supress these limitations of PCA and LDA and utilizing their strengths, in this paper, PCA + LDA has been adopted, where PCA algorithm is applied to reduce the dimensionality of large features while LDA algorithm is applied on PCA weights to increase the separability between the classes.

\subsection{Matching module and normalization score}

In the matching module of the proposed system, the Nearest Neighbor Classifier that uses the cosine Mahalanobis distance has been used.The criterion for similarity or dissimilarity is to minimize the distance (score) between the input query sample and the stored template. Assume that two vectors $\mathrm{Vi}$ and $\mathrm{Vj}$ stands for the feature vectors of query and template in the database images, respectively. Then the distance between $\mathrm{V}_{\mathrm{i}}$ and $\mathrm{Vj}$ is calculated by the following equation:

$d_{M a}\left(V_{i}, V_{j}\right)=\left(V_{i}-V_{j}\right)^{T} C^{-1}\left(V_{i}-V_{j}\right)$

where $\mathrm{C}$ refers to the covariance matrix. Prior to finding the decision, a method named Min-Maxnormalization scheme is employed to transform the matching score into [0 1]. Given a set of matching scores $\left\{X_{k}\right\}$, where $K=1,2, \ldots$, n. The normalization scores are given as:

$X_{K}^{\prime}=\frac{X_{K}-\min }{\max -\min }$

where $X_{K}^{\prime}$ represents the normalized scores. The final decision is made using normalized score.

\section{Experiments}

In this section, we present an experimental evaluation of the proposed FKP person authentication system based on Bank BSIF descriptor.

(b)

(a)
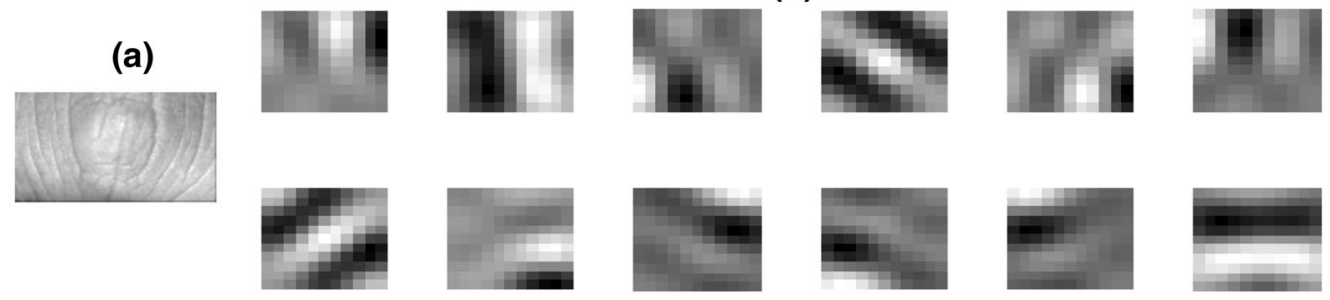

(c)
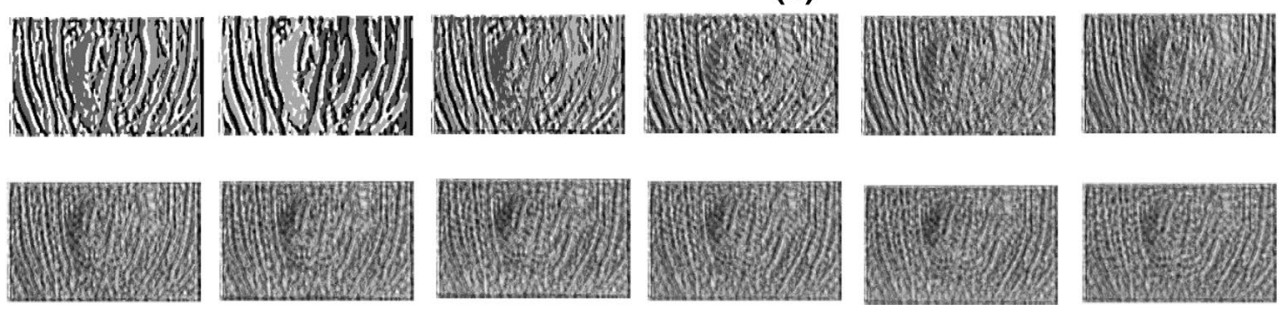

(d)
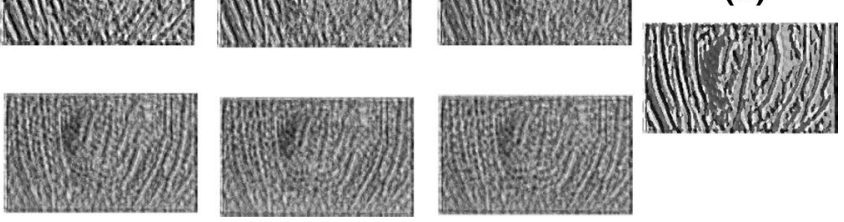

Fig. 3 a Sample of the FKP ROI image b BSIF filter with a size $11 \times 11$ and of length $12 \mathbf{c}$ BSIF features d final BSIF 


\subsection{Database}

The proposed system has been tested on the publicly available FKP dataset that provided by Hong Kong Polytechnic University (PolyU) (PolyU 2010). This database has 7920 images collected from 165 persons with 125 males and 40 females, their age is in the range of [20-50] years old. The images were captured in two sessions, with 48 different FKP images ofeach individual. Four finger types of every person have been collected that are: left index (LIF), left middle (LMF), right index (RIF) and right middle (RMF). Every finger type has 6 images in each session. There are total 1980 number of images for each finger type.

\subsection{Experimental protocol}

The evaluation of biometric recognition systems can be done in two modes: identification and verification. For the identification mode, the results have been presented in the form of the recognition rate. Rank-1 that is given by:

Rank $-1=\frac{\mathrm{N}_{\mathrm{i}}}{\mathrm{N}} \times 100(\%)$

where $\mathrm{N}_{\mathrm{i}}$ denotes the number of images successfully assigned to the right identity and $\mathrm{N}$ stands for the overall number of images attempts to be identified. The cumulative match curves (CMC) is used in closed-set identification task. The CMC present the accuracy performance of a biometric system and shows how frequently the individual's template appears in the ranks based on the match rates (Jain et al. 2007). Thus, we have also adopted CMC curve in the experimental results. For the verification mode, we present the results in the form of the error equal rate (EER), when FAR (false accept rate) equal FRR (false reject rate). Moreover, to visually depict the performance of the biometric system, Receiver Operating Characteristics (ROC) curves have also been reported. A ROC curve explains how the FAR values are changed compared to the values of the Genuine Acceptance Rate (GAR) values.In addition in the mode of verification the measures VR@1\%FAR (i.e., verification rate at operating point of $1 \%$ FAR), VR $@ 0.1 \%$ FAR and VR@0.01\%FAR have been used. Namely, VR@0.1\% FAR refers to the verification rate that is equivalent to $1 \%$ FRR, is calculated for a FAR equal to $0.1 \%$. This operating point is widely adopted in the biometric research community specially when the number of comparisons for "inter-class" tests (or "imposter tests") is more than 1000. The verification rate is significant for studying the behavior of systems with low FAR using a large database or for simulating large scale application in order to improve system's security and performance.

\subsection{Experiment results}

Here, we report three different experiments: Experiment I-construction of bank of BSIF filters, Experiment IIcomparaisons between BSIF and B-BSIF and with the existing FKP recognition systems, and Experiment IIImultibiometric FKP recognition system.

\subsubsection{Experiment I: construction of bank of BSIF filters}

The goal of this experiment is to construct a bank of filters BSIF. For comprehensive analysis, each individual BSIF filter with different parameter combination is applied on each individual FKP modality (LIF, LMF, RIF and RMF) in order to select the best BSIF parameters and respective filters. Since, the filter parameters (filter size (k) and filter length (n)) have a great influence on the performance of the proposed system, several sub-experiments were performed in both FKP identification and verification modes. The results are reported in Table 1. In Table 1, it is easy to see that the performance increases with the increase of the length (n) of the BSIF descriptor. The parameters $(\mathrm{k}$, n) combinations that achieve high ranking performance on single modality has been selected and used to build the bank of BSIF (B-BSIF). These parameters are presented in Table 2, which are at this stage been fixed and used as estimated parameters for subsequent experiments. The model of the B-BSIF descriptor is illustrated in Fig. 4. As can be notice, the bank is composed of different BSIF descriptor sizes, which are $(17 \times 17),(15 \times 15),(13 \times 13),(11 \times 11)$, $(9 \times 9)$ and $(7 \times 7)$ with the length of 12 bits. This bank of filters is applied to the each input FKP trait, as Fig. 4 also representates an example of using B-BSIF.

\subsubsection{Experiment II: comparisons between BSIF and B-BSIF and with the existing FKP recognition systems}

Here, we present two sets of experiments. First, experimental analysis to show efficacy of B-BSIF in comparsion with BSIF image texture descriptors for FKP based person recognition. The second experimentsproviding a comparison of the proposed system with prior works in the literatures. In this sets of experiments, the best BSIF parameters estimated in experiment $I$ is utilized to built B-BSIF descriptor. The results of BSIF and B-BSIF on individual FKP traits are presented in Table 3. We can observe in Tables 1 and 3 that B-BSIF performs better than BSIF. The proposed method using B-BSIF achieves higher accuracy on the LMF,RMF, and LIF, modalities, e.g., on RMF modality the system achieved EER $=0.03 \%$ 
Table 1 The all parameters BSIF applied on the single finger

\begin{tabular}{|c|c|c|c|c|c|c|c|c|c|}
\hline \multicolumn{2}{|c|}{ Parameters } & \multicolumn{2}{|l|}{ LMF } & \multicolumn{2}{|l|}{ LIF } & \multicolumn{2}{|l|}{ RIF } & \multicolumn{2}{|l|}{ RMF } \\
\hline $\mathrm{k}$ & $n$ & Rank-1 (\%) & $\operatorname{EER}(\%)$ & Rank-1 (\%) & $\operatorname{EER}(\%)$ & Rank-1 (\%) & $\operatorname{EER}(\%)$ & Rank-1 (\%) & $\operatorname{EER}(\%)$ \\
\hline $17 \times 17$ & 12 & 98.08 & 0.30 & 97.88 & 0.30 & 96.57 & 0.51 & 98.08 & 0.20 \\
\hline $17 \times 17$ & 11 & 95.56 & 0.89 & 95.66 & 0.91 & 95.05 & 0.91 & 95.96 & 0.51 \\
\hline $17 \times 17$ & 10 & 87.98 & 2.02 & 87.68 & 2.22 & 87.27 & 3.03 & 89.60 & 2.21 \\
\hline $17 \times 17$ & 9 & 83.54 & 2.93 & 83.94 & 3.23 & 81.11 & 4.76 & 84.04 & 3.64 \\
\hline $17 \times 17$ & 8 & 79.49 & 4.27 & 81.41 & 4.04 & 77.27 & 5.13 & 77.88 & 5.13 \\
\hline $15 \times 15$ & 12 & 98.28 & 0.22 & 98.38 & 0.30 & 97.58 & 0.61 & 98.89 & 0.10 \\
\hline $15 \times 15$ & 11 & 96.36 & 0.81 & 96.97 & 0.59 & 95.35 & 0.79 & 96.57 & 0.38 \\
\hline $15 \times 15$ & 10 & 89.80 & 2.02 & 89.29 & 2.02 & 88.79 & 2.22 & 89.70 & 1.54 \\
\hline $15 \times 15$ & 9 & 84.65 & 3.12 & 81.72 & 3.13 & 81.62 & 4.14 & 85.15 & 2.53 \\
\hline $15 \times 15$ & 8 & 80.81 & 3.74 & 81.82 & 4.15 & 82.12 & 3.52 & 80.81 & 3.64 \\
\hline $13 \times 13$ & 12 & 98.08 & 0.20 & 98.48 & 0.30 & 97.27 & 0.40 & 98.59 & 0.20 \\
\hline $13 \times 13$ & 11 & 96.46 & 0.41 & 97.07 & 0.51 & 96.16 & 0.61 & 97.37 & 0.51 \\
\hline $13 \times 13$ & 10 & 91.62 & 1.21 & 90.61 & 1.62 & 87.88 & 2.41 & 90.40 & 2.12 \\
\hline $13 \times 13$ & 9 & 84.95 & 2.44 & 86.16 & 2.52 & 84.14 & 3.84 & 85.45 & 2.83 \\
\hline $13 \times 13$ & 8 & 82.63 & 3.85 & 83.94 & 3.13 & 82.93 & 3.54 & 84.14 & 3.44 \\
\hline $11 \times 11$ & 12 & 98.79 & 0.20 & 98.89 & 0.20 & 97.68 & 0.40 & 98.89 & 0.10 \\
\hline $11 \times 11$ & 11 & 96.46 & 0.30 & 97.37 & 0.40 & 96.06 & 0.81 & 97.17 & 0.30 \\
\hline $11 \times 11$ & 10 & 90.51 & 1.92 & 91.52 & 1.72 & 87.68 & 2.42 & 92.53 & 1.62 \\
\hline $11 \times 11$ & 9 & 85.96 & 2.63 & 86.97 & 2.83 & 83.74 & 3.94 & 86.06 & 2.92 \\
\hline $11 \times 11$ & 8 & 85.35 & 2.93 & 85.45 & 2.91 & 83.84 & 3.33 & 88.79 & 2.31 \\
\hline $9 \times 9$ & 12 & 98.48 & 0.30 & 98.89 & 0.18 & 97.68 & 0.40 & 98.79 & 0.40 \\
\hline $9 \times 9$ & 11 & 96.97 & 0.69 & 97.68 & 0.38 & 94.44 & 0.99 & 97.07 & 0.51 \\
\hline $9 \times 9$ & 10 & 89.70 & 1.70 & 87.58 & 1.92 & 86.06 & 2.85 & 89.49 & 1.92 \\
\hline $9 \times 9$ & 9 & 85.76 & 2.22 & 85.66 & 2.10 & 81.72 & 3.94 & 87.98 & 2.61 \\
\hline $9 \times 9$ & 8 & 86.26 & 3.02 & 87.78 & 2.51 & 83.33 & 3.85 & 88.38 & 2.53 \\
\hline $7 \times 7$ & 12 & 96.46 & 0.51 & 97.37 & 0.38 & 95.86 & 0.62 & 97.17 & 0.61 \\
\hline $7 \times 7$ & 11 & 94.85 & 0.81 & 96.16 & 0.71 & 92.93 & 1.01 & 95.15 & 1.01 \\
\hline $7 \times 7$ & 10 & 84.14 & 3.34 & 83.13 & 2.83 & 80.91 & 3.33 & 84.14 & 84.14 \\
\hline $7 \times 7$ & 9 & 81.82 & 3.93 & 77.37 & 4.05 & 78.08 & 4.75 & 79.60 & 4.34 \\
\hline $7 \times 7$ & 8 & 85.25 & 3.33 & 81.21 & 3.52 & 79.19 & 3.94 & 84.95 & 3.74 \\
\hline $5 \times 5$ & 12 & 88.18 & 2.32 & 89.39 & 1.41 & 87.47 & 2.63 & 89.90 & 1.90 \\
\hline $5 \times 5$ & 11 & 82.12 & 3.21 & 80.81 & 3.23 & 80.30 & 3.45 & 83.94 & 3.33 \\
\hline $5 \times 5$ & 10 & 58.69 & 8.48 & 57.17 & 8.44 & 59.29 & 8.38 & 64.24 & 7.78 \\
\hline $5 \times 5$ & 9 & 60.91 & 8.92 & 55.35 & 8.36 & 58.79 & 9.07 & 60.51 & 8.49 \\
\hline $5 \times 5$ & 8 & 69.09 & 7.27 & 65.86 & 7.07 & 66.57 & 7.98 & 67.78 & 6.66 \\
\hline
\end{tabular}

Table 2 The best BSIF parameters of the bank BSIF

\begin{tabular}{ll}
\hline Parameters & \\
\hline $\mathrm{k}$ & $n$ \\
\hline $17 \times 17$ & 12 \\
$15 \times 15$ & 12 \\
$13 \times 13$ & 12 \\
$11 \times 11$ & 12 \\
$9 \times 9$ & 12 \\
$7 \times 7$ & 12 \\
\hline
\end{tabular}

and Rank-1 accuracy $=99.70 \%$ in verification and identification modes, respectively.

The BSIF and B-BSIF comparsion results are also presented in term of CMC and ROC curves, which can be seen in Figs. 5, 6, 7, 8. These graphs also reports comparison study between the (BSIF + PCA + LDA) and the proposed method (B-BSIF + PCA + LDA) using FKP modality. The results clearly show that the performance is higher when the system uses the features of the B-BSIF descriptor.

To further demonstrate the efficacy of the proposed system, the comparison of the proposed system with prior works in the literatures on individual FKP modalities is 
Fig. 4 The model of the B-BSIF descriptor: a the input ROI FKP trait, $\mathbf{b}$ the results of applying the different BSIF descriptor (BSIF code images), and $\mathbf{c}$ the histograms of the BSIF code images

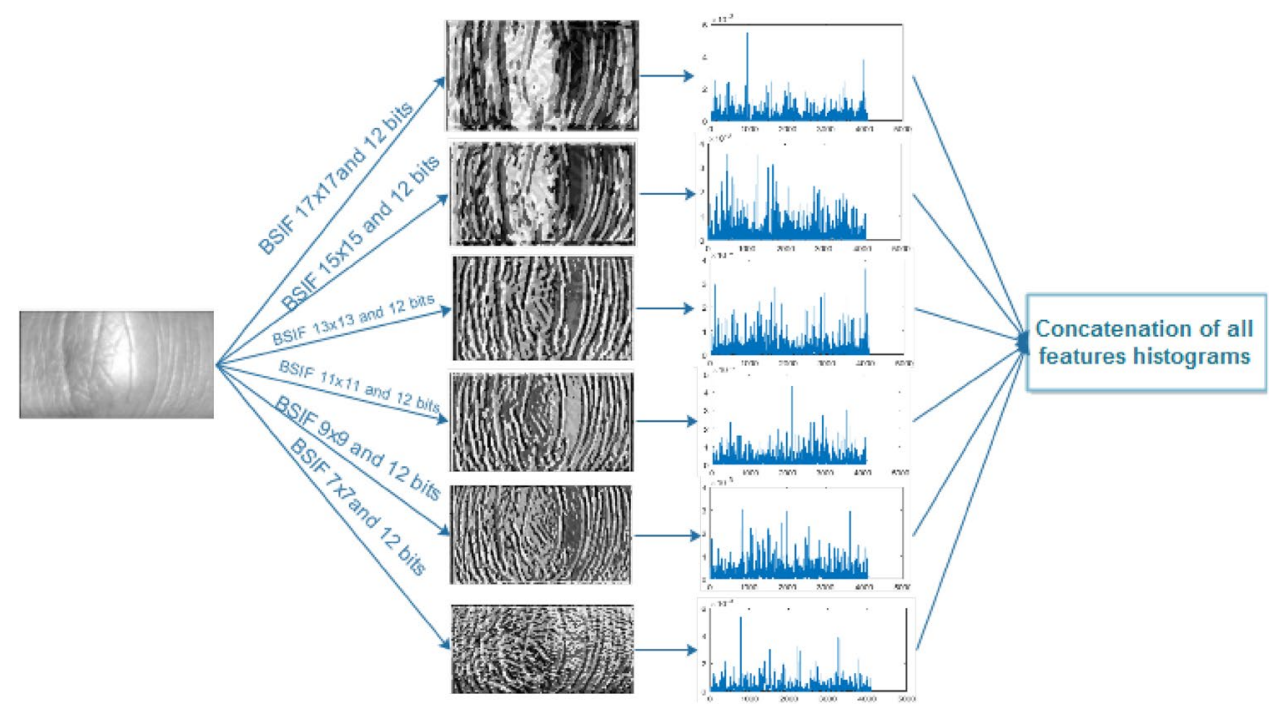

Table 3 The results B-BSIF on single modalities

\begin{tabular}{lllcll}
\hline Modality & Identification & \multicolumn{2}{l}{ Verification } \\
\cline { 3 - 6 } & Rank-1 $(\%)$ & EER $(\%)$ & VR@ 1\%FAR $(\%)$ & $\begin{array}{l}\text { VR@ 0.1\%FAR } \\
(\%)\end{array}$ & $\begin{array}{l}\text { VR@0.01\%FAR } \\
(\%)\end{array}$ \\
\hline LMF & 99.70 & 0.10 & 100.00 & 99.90 & 99.19 \\
LIF & 99.70 & 0.11 & 99.90 & 99.80 & 99.29 \\
RIF & 98.69 & 0.40 & 99.80 & 99.29 & 98.18 \\
RMF & 99.70 & 0.03 & 100.00 & 100.00 & 99.49 \\
\hline
\end{tabular}
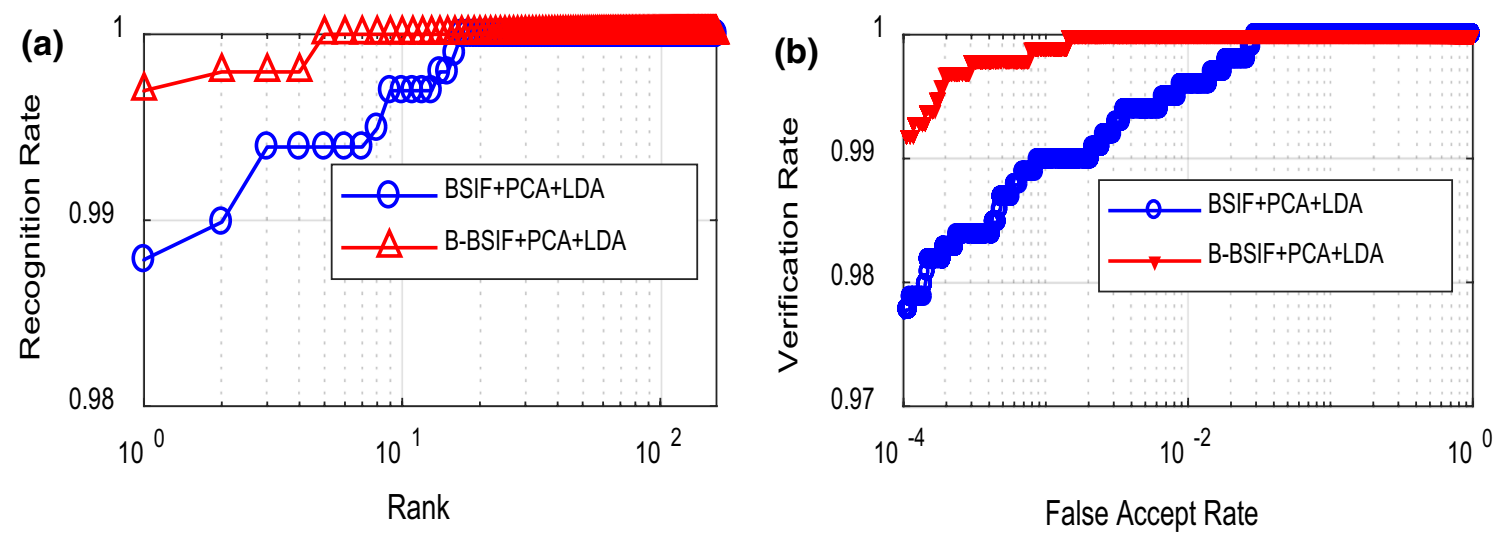

Fig. 5 Comparison between BSIFand B-BSIF features for LMF modality a CMC curves b ROC curves

given in Table 4 . The proposed scheme outperforms the existing FKP person recognition system. For instance, the proposed method on RMF modality is able to attain $99.70 \%$ and $0.03 \%$ accuracy and error rate in identification and verification scenario, respectively. While, FKP based person recognition systems proposed in (Shariatmadar and Faez 2013) achieved $96.72 \%$ and $0.354 \%$ accuracy and error rate in identification and verification scenario, respectively.

\subsubsection{Experiment III: Multibiometric FKP recognition system}

The aim of this experiment is to study performance of the system in the case of information fusion, since multimodal systems fusing information from different sources are able to alleviate limitations (e.g., accuracy, noise, etc.) of the unimodal biometric systems. Thus, we studied different scenarios when the information presented by different finger 

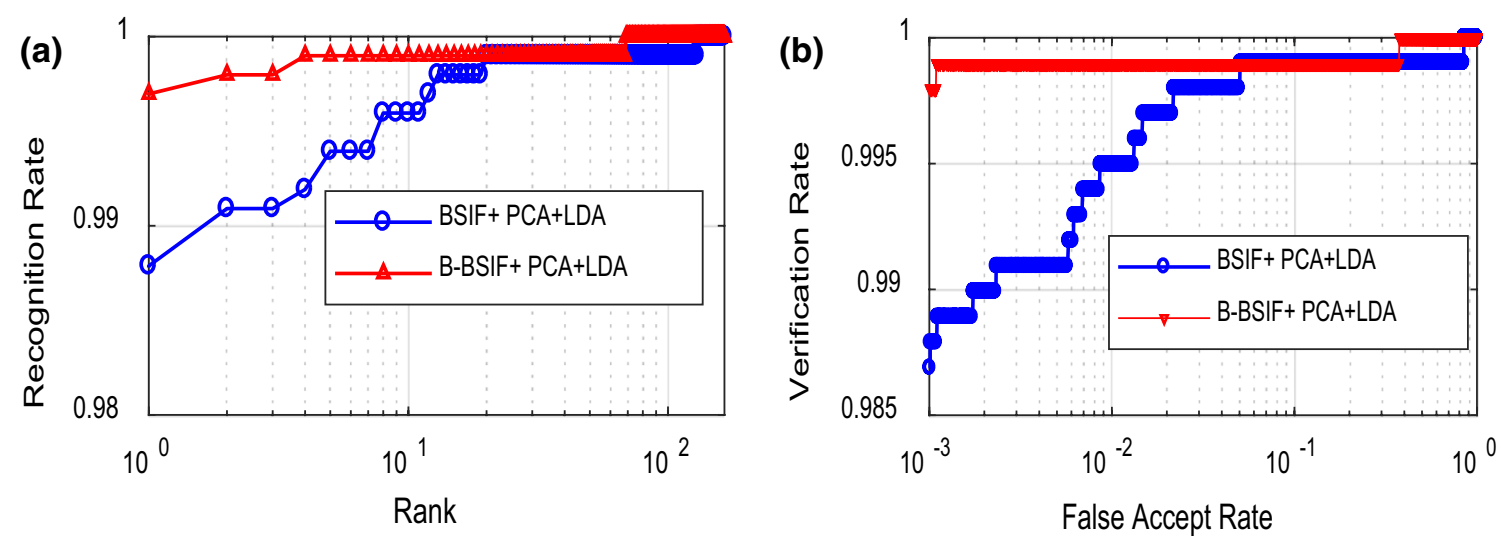

Fig. 6 Comparison between BSIF and B-BSIF features for LIF modality a CMC curves b ROC curves
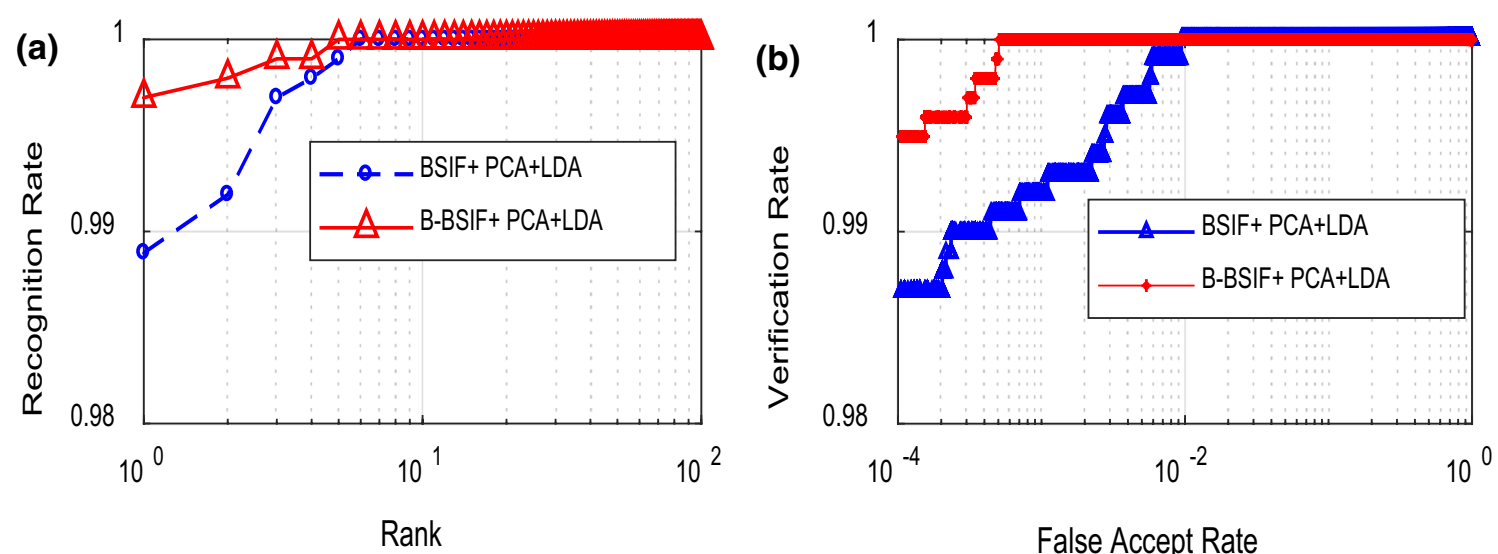

False Accept Rate

Fig. 7 Comparison between BSIF and B-BSIF features for RMF modality a CMC curves b ROC curves
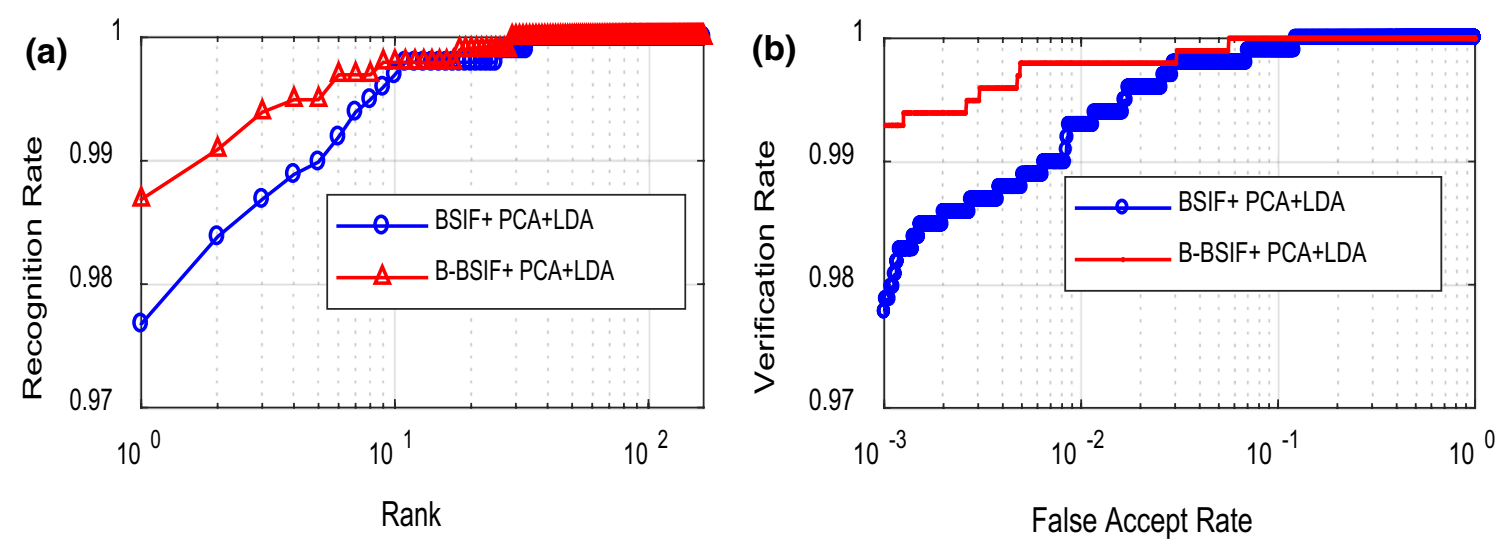

Fig. 8 Comparison between BSIF and B-BSIF features for RIF modality a CMC curves $\mathbf{b}$ ROC curves

types (LIF, LMF, RIF, and RMF) modality are integrated. The information were fused at score level using sum and min fusion rules. Four distinct experimental investigation were conducted by fusing only two types of fingers
(Table 5), three types fingers (Table 6), all types of fingers (Table 7) and comparsion with existing FKP multimodal methods (Table 8). In Tables 5, 6 and 7, we can see that the multimodal FKP systems that fuse information from 
Table 4 Comparative of proposed personal recognition method with the existing approaches for FKP using PolyU dataset

\begin{tabular}{llllll}
\hline Identification (rank-1) & & & & \\
\hline Reference & Year & LMF (\%) & LIF (\%) & RIF (\%) & RMF (\%) \\
\hline El-Tarhouni et al. (2014) & 2014 & 94.70 & 93.80 & 92.20 & 94.80 \\
Shariatmadar and Faez (2013) & 2013 & 95.54 & 94.33 & 95.93 & 96.72 \\
Zeinali et al. (2014) & 2014 & 90.30 & 88.68 & 89.79 & 89.79 \\
Our paper & - & 99.70 & 99.70 & 98.69 & 99.70 \\
Verification (EER) & & & & & \\
Kong et al. (2014) & 2014 & 0.15 & 0.26 & 0.20 & 0.24 \\
Shariatmadar and Faez (2013) & 2013 & 0.384 & 0.54 & 0.781 & 0.354 \\
Shariatmadar and Faez (2014) & 2014 & 0.29 & 0.267 & 0.284 & 0.25 \\
Nigam et al. (2016) & 2016 & 1.009 & 1.077 & 0.740 & 1.0615 \\
Morales et al. (2011) & 2011 & 0.43 & 1.02 & 0.95 & 0.91 \\
Our paper & - & 0.10 & 0.11 & 0.40 & 0.03 \\
\hline
\end{tabular}

Table 5 EER/ROR (\%) obtained by the fusion of two types of fingers

\begin{tabular}{|c|c|c|c|c|c|c|}
\hline \multirow{3}{*}{$\begin{array}{l}\text { EER/ROR (\%) Auth/ } \\
\text { Ident }\end{array}$} & \multicolumn{6}{|c|}{ Fusion of two types of fingers } \\
\hline & \multicolumn{2}{|l|}{ LIF-LMF } & \multicolumn{2}{|c|}{ LMF-RMF } & \multicolumn{2}{|l|}{ LMF-RIF } \\
\hline & $\operatorname{EER}(\%)$ & ROR (\%) & $\operatorname{EER}(\%)$ & ROR (\%) & $\operatorname{EER}(\%)$ & ROR (\%) \\
\hline Sum rule & 0.00 & 100.00 & 0.00 & 99.90 & 0.08 & 99.90 \\
\hline Min rule & 0.02 & 99.90 & 0.09 & 99.90 & 0.10 & 99.90 \\
\hline \multirow{2}{*}{$\begin{array}{l}\text { EER/ROR (\%) Auth/ } \\
\text { Ident }\end{array}$} & \multicolumn{2}{|l|}{ RIF-RMF } & \multicolumn{2}{|l|}{ LIF-RIF } & \multicolumn{2}{|l|}{ RMF-LIF } \\
\hline & $\operatorname{EER}(\%)$ & ROR (\%) & $\operatorname{EER}(\%)$ & ROR (\%) & $\operatorname{EER}(\%)$ & ROR (\%) \\
\hline Sum rule & 0.09 & 99.80 & 0.00 & 100.00 & 0.00 & 100.00 \\
\hline Min rule & 0.10 & 99.80 & 0.00 & 100.00 & 0.00 & 100.00 \\
\hline
\end{tabular}

Table 6 EER/ROR (\%) obtained by the fusion of three types of fingers

\begin{tabular}{|c|c|c|c|c|c|c|c|c|}
\hline \multirow{3}{*}{$\begin{array}{l}\text { EER/ROR } \\
\text { (\%) Auth/ } \\
\text { Ident }\end{array}$} & \multicolumn{8}{|c|}{ Fusion of three types of fingers } \\
\hline & \multicolumn{2}{|c|}{ LMF-LIF-RIF } & \multicolumn{2}{|c|}{ LMF-LIF-RMF } & \multicolumn{2}{|c|}{ RMF-RIF-LMF } & \multicolumn{2}{|c|}{ RMF-RIF-LIF } \\
\hline & $\operatorname{EER}(\%)$ & ROR (\%) & $\operatorname{EER}(\%)$ & ROR $(\%)$ & $\operatorname{EER}(\%)$ & $\operatorname{ROR}(\%)$ & $\operatorname{EER}(\%)$ & ROR $(\%)$ \\
\hline Sum rule & 0.00 & 100.00 & 0.00 & 100.00 & 0.01 & 99.90 & 0.00 & 100.00 \\
\hline Min rule & 0.00 & 100.00 & 0.00 & 100.00 & 0.10 & 99.90 & 0.00 & 100.00 \\
\hline
\end{tabular}

more than one source improves the accuracy of unimodal systems. For example, using only LIF modality features for person authentication (Table 4) resulted into $0.11 \%$ (EER) and $99.70 \%$ (rank-1 accuracy), while sum fusion of LIF and RMF (Table 5) yielded $0.00 \%$ (EER) and 100\% (rank-1 accuracy).

The comparison of proposed multimodal system with existing systems in the literature is presented in Table 8 . We can notice in Table 8 that the proposed system under verification mode attained lowest EER\% compared to EER\%by systems in (Shariatmadar and Faez 2014) and (Morales et al. 2011). In identification mode, the proposed system achieved highest recognition rate compared to schemes designed in (Shariatmadar and Faez 2013) and (Zeinali et al. 2014).
Table 7 EER/ROR (\%) obtained by the fusion of all fingers

\begin{tabular}{lll}
\hline EER/ROR (\%) Auth/Ident & \multicolumn{2}{l}{ All fingers } \\
\cline { 2 - 3 } & EER (\%) & ROR (\%) \\
\hline Sum rule & 0.00 & 100.00 \\
Min rule & 0.00 & 100.00 \\
\hline
\end{tabular}

\section{Conclusion}

This paper presents a new method for recognizing individuals based on their finger knuckle patterns (FKP). Specifically, the proposed method uses bank of mutli-scale 
Table 8 Comparative of the proposed multimodal system with the existing approaches for FKP

\begin{tabular}{lllll}
\hline References & & LIF-RIF & LIF-LMF & Four fingers \\
\hline Shariatmadar and Faez (2014) & EER (\%) & 0.162 & 0.125 & 0.00 \\
& Rank-1 (\%) & - & - & - \\
Morales et al. (2011) & EER (\%) & 0.08 & 0.04 & 0.00 \\
Shariatmadar and Faez (2013) & Rank-1 (\%) & - & - & - \\
& EER (\%) & 0.162 & 0.125 & 0.00 \\
Zeinali et al. (2014) & Rank-1 (\%) & 98.69 & 99.09 & 100.00 \\
& EER (\%) & - & - & - \\
Proposed system & Rank-1 (\%) & 97.27 & 95.55 & 99.29 \\
& EER (\%) & 0.00 & 0.00 & 0.00 \\
& Rank-1 (\%) & 100.00 & 100.00 & 100.00 \\
\hline
\end{tabular}

binarized statistical image features (B-BSIF). The system first extracts features using different best performing convolution filters to encode FKP trait. Then, histograms extracted from the encoded FKP images are concatenated into a big feature vector. A PCA + LDA technique is used to reduce the dimensionality of the feature vector. Finally, the nearest neighbor classifier, based on cosine Mahalanobis distance is used to recognition the user. The experimental results on a publicly available FKP PolyU database shows not only that the FKP modality is quite useful in biometric systems, presented B-BSIF scheme is very powerful and effective in FKP recognition but also the proposed mechanism outperform other existing state-ofthe-art techniques. Furthermore, reported experiments also show that use of multiple finger types (i.e., multimodal system) improved the performance of systems using only single finger FKP information. In the future, to improve the security and robustness of the system against spoofing we aim to fuse FKP modality with other modalities (e.g., iris or voice) on mobile devices.

\section{References}

Adeoye OS (2010) A survey of emerging biometric technologies. Int J Comput Appl 9(10):1-5

Akhtar Z, Alfarid N (2011) Secure learning algorithm for multimodal biometric systems against spoof attacks. In: Proc. international conference on information and network technology (IPCSIT), vol. 4, pp 52-57

Akhtar Z, Fumera G, Marcialis GL, Roli F (2011) Robustness evaluation of biometric systems under spoof attacks. In: International conference on image analysis and processing, pp 159-168

Angelov PP, Gu X (2018) Deep rule-based classifier with human-level performance and characteristics. Inf Sci (Ny) 463-464:196-213

Bao R-J, Rong H-J, Angelov PP, Chen B, Wong PK (2018) Correntropy-based evolving fuzzy neural system. IEEE Trans Fuzzy Syst 26(3):1324-1338

Belhumeur PN, Hespanha JP, Kriegman DJ (1997) Eigenfaces vs. fisherfaces: recognition using class specific linear projection. IEEE Trans Pattern Anal Mach Intell 19(7):711-720
Chaa M, Boukezzoula N, Meraoumia A (2018) Features-level fusion of reflectance and illumination images in finger-knuckle-print identification system. Int J Artif Intell Tools 27(3):1850007

Chlaoua R, Meraoumia A, Aiadi KE, Korichi M (2018) Deep learning for finger-knuckle-print identification system based on PCANet and SVM classifier. Evol Syst. https://doi.org/10.1007/s 1253 0-018-9227-y

El-Tarhouni W, Shaikh MK, Boubchir L, Bouridane A (2014) Multiscale shift local binary pattern based-descriptor for fingerknuckle-print recognition. In: 26th International Conference on Microelectronics (ICM), 2014, pp 184-187

Jain AK, Flynn P, Ross AA (2007) Handbook of biometrics. Springer, Berlin

Kannala J, Rahtu E (2012) Bsif: binarized statistical image features. In: 21st International Conference on Pattern Recognition (ICPR), 2012, pp 1363-1366

Kong T, Yang G, Yang L (2014) A hierarchical classification method for finger knuckle print recognition. EURASIP J Adv Signal Process 2014(1):44

Morales A, Travieso CM, Ferrer MA, Alonso JB (2011) Improved finger-knuckle-print authentication based on orientation enhancement. Electron Lett 47(6):380-381

Nigam A, Tiwari K, Gupta P (2016) Multiple texture information fusion for finger-knuckle-print authentication system. Neurocomputing 188:190-205

PolyU (2010) The Hong Kong polytechnic university (PolyU) FingerKnuckle-Print Database [Online]. http://www.comp.polyu.edu.hk/ biometrics/FKP.html

Rani E, Shanmugalakshmi R (2013) Finger knuckle print recognition techniques-a survey. Int J Eng Sci 2(11):62-69

Shariatmadar ZS, Faez K (2013) Finger-knuckle-print recognition via encoding local-binary-pattern. J Circuits Syst Comput 22(6): 1350050

Shariatmadar ZS, Faez K (2014) Finger-Knuckle-Print recognition performance improvement via multi-instance fusion at the score level. Opt J Light Electron Opt 125(3):908-910

Turk M, Pentland A (1991) Eigenfaces for recognition. J Cogn Neurosci 3(1):71-86

Zeinali B, Ayatollahi A, Kakooei M (2014) "A novel method of applying directional filter bank (DFB) for finger-knuckle-print (FKP) recognition. In: 22nd Iranian Conference on Electrical engineering (ICEE), 2014, pp 500-504

Zhai Y et al. (2018) A novel finger-knuckle-print recognition based on batch-normalized CNN. In: Chinese conference on biometric recognition, pp 11-21

Zhang L, Li H (2012) Encoding local image patterns using Riesz transforms: With applications to palmprint and finger-knuckle-print recognition. Image Vis Comput 30(12):1043-1051 
Zhang L, Zhang L, Zhang D, Zhu H (2010) Online finger-knuckleprint verification for personal authentication. Pattern Recognit 43(7):2560-2571

Zhang L, Zhang L, Zhang D, Zhu H (2011) Ensemble of local and global information for finger-knuckle-print recognition. Pattern Recognit 44(9):1990-1998

Zhang D, Lu G, Zhang L (2018a) Finger-knuckle-print verification with score level adaptive binary fusion. In: Advanced Biometrics. Springer, Cham, pp 151-174
Zhang D, Lu G, Zhang L (2018b) Finger-knuckle-print verification. In: Advanced biometrics. Springer, Cham, pp 85-109

Publisher's Note Springer Nature remains neutral with regard to jurisdictional claims in published maps and institutional affiliations. 